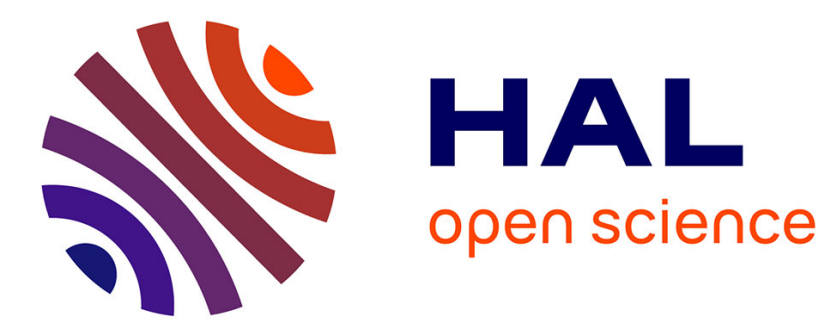

\title{
Transitions de radiofréquence à deux quanta et effet Raman
}

\author{
A. Kastler
}

\section{To cite this version:}

A. Kastler. Transitions de radiofréquence à deux quanta et effet Raman. J. Phys. Radium, 1954, 15

(4), pp.323-324. 10.1051/jphysrad:01954001504032301 • jpa-00234924

\section{HAL Id: jpa-00234924 https://hal.science/jpa-00234924}

Submitted on 1 Jan 1954

HAL is a multi-disciplinary open access archive for the deposit and dissemination of scientific research documents, whether they are published or not. The documents may come from teaching and research institutions in France or abroad, or from public or private research centers.
L'archive ouverte pluridisciplinaire HAL, est destinée au dépôt et à la diffusion de documents scientifiques de niveau recherche, publiés ou non, émanant des établissements d'enseignement et de recherche français ou étrangers, des laboratoires publics ou privés. 


\title{
TRANSITIONS DE RADIOFRÉQUENGE A DEUX QUANTA ET EFFET RAMAN
}

\author{
Par A. KASTLER,
}

Laboratoire de Physique E. N. S., Paris (France).

La méthode de résonance électrique des jets moléculaires, développée par l'équipe de Rabi à Columbia University a permis à Grabner et Hughes [1] de caractériser dans le cas de la molécule ${ }^{8} \cdot \mathrm{RbF}$ des transitions spectrales faisant intervenir deux quanta électromagnétiques. La transition observée correspond au passage entre deux niveaux d'énergie de la structure hyperfine due au moment électrique quadrupolaire du noyau ${ }_{85} \mathrm{Rb}$, niveaux caractérisés par les nombres quantiques

$$
J=\mathrm{l}, \quad F_{1}=\frac{3}{2} \quad \text { et } \quad J=\mathrm{I}, \quad F_{1}=\frac{7}{2} .
$$

Le saut correspondant du nombre quantique $F_{1}=J+I_{1}\left(J\right.$, quantum de rotation; $I_{1}$, spin nucléaire de ${ }_{55} \mathrm{Rb}$ ) est un saut de deux unités : $\Delta F_{1}=2$. Cette transition se fait en absence de champ électrique constant, sous l'influence simultanée de deux champs électriques de fréquences différentes tel que la somme des fréquences corresponde à l'intervalle énergétique de la transition :

$$
v_{1}+v_{2}=\frac{\Delta E}{h}=6,328 M h_{2} .
$$

- On peut, comme l'a suggéré Rabi, interpréter la transition par le concours de deux quanta électromagnétiques $h \vee_{1}$ et $h \vee_{2}$ dont les énergies s'ajoutent pour réaliser le saut $\Delta E$ entre les niveaux d'énergie de la molécule (fig. I). Un tel processus peut être rapproché d'autres phénomènes taisant intervenir deux quanta d'énergie rayonnante. Il en est ainsi dans l'effet Raman, c'est-à-dire dans la diffusion moléculaire de la lumière avec changement de fréquence. Nous savons que l'acte de diffusion peut être décrit par le mécanisme de Smékal [2] : absorption d'un 
quantum lumineux incident $h v_{1}$ à partir du niveau fondamental $E_{k}$ suivi de la réémission d'un quantum diffusé $h v_{2}$ amenant la molécule à un état vibratoire



Fig. 1 .



Fig. 2.

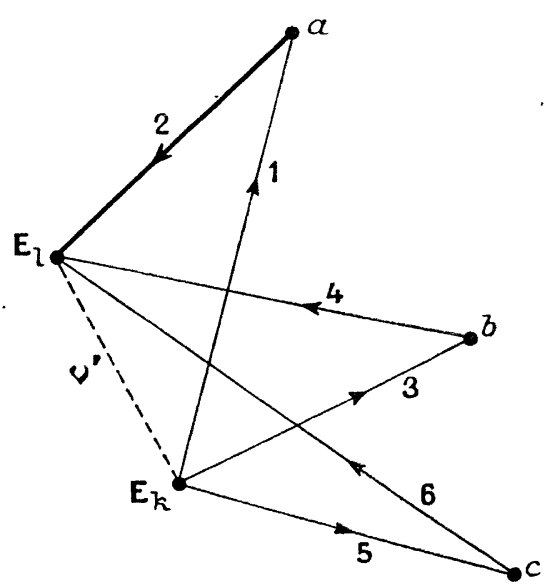

Fig. 3. excité $E_{l}$. La fréquence Raman $v^{\prime}$ qui correspond à l'intervalle est égale à la différence $: v^{\prime}=v_{1}-v_{2}$. Ici aussi il y a donc intervention de deux quanta électromagnétiques pour réaliser la transition, mais suivant que le " niveau intermédiaire " se trouve entre les niveaux $E_{l}$ et $E_{k}$ (transition hertzienne) ou en dehors de leur intervalle (effet Raman), cet intervalle apparaîtra comme somme ou comme différence des deux quanta d'énergie rayonnante. L'ensemble des divers mécanismes possibles avaient été discuté par Kramers et Heisenberg dans leur Mémoire fondamental conduisant à la formule de dispersion [3]. La figure 3, extraite de ce Mémoire, résume les divers cas possibles de transitions faisant intervenir deux quanta électromagnétiques, et conduisant d'un état d'énergie $E_{k}$ à un état supérieur $\boldsymbol{E}_{l}$. Ce rapprochement suggère les remarques suivantes :

Io Il y a lieu de chercher si la transition de la molécule $\mathrm{Rb} F$ du domaine hertzien peut être réalisée également par deux oscillateurs de fréquences hertziennes $v_{1}$ et $v_{2}$ telles que leur différence corresponde à l'intervalle énergétique :

$$
v_{1}-v_{2}=\frac{\Delta E}{h}
$$

$2^{\circ}$ Le bilan de moment cinétique entre champ électromagnétique et molécule doit fournir dans les deux cas les règles de polarisation régissant les transitions à deux quanta. En particulier l'intervention de deux quanta permet une variation de $2 \hbar \mathrm{du}$ moment cinétique de la molécule (saut $\Delta J=2$ dans l'effet Raman, saut $\Delta \boldsymbol{F}=2$ dans le cas de $\mathrm{Rb} F$ ). La règle de polarisation correspondante peut s'énoncer ainsi :

Dans le cas d'une transition additive $\left(v_{1}+v_{2}=\frac{\Delta E}{h}\right)$

l'intensité de la transition est grande lorsque les deux radiations ont même sens de polarisation circulaire, dans le cas d'une transition soustractive $\left(v_{1}-v_{2}=\frac{\Delta E}{h}\right)$ la transition n'est intense que si les deux radiations ont des polarisations circulaires inverses. Cette règle de polarisation a été mise en évidence en spectrographie Raman (inversion de polarisation circulaire des raies Raman $\Delta J=2$, effet Hanle-Bär [4]. Il serait intéressant de vérifier la règle de polarisation dans le domaine hertzien en employant des champs électriques tournants à la place de champs oscillants rectilignes.

\section{BIBLIOGRAPHIE.}

[1] Grabner L. et Hughes H. - Phys. Rev., i95o, 79, 3 I 4 et $829 ; 1951,82,56$ I.

[2] Smekal A. - Z. Physik, 1925, 32, $241 ; 34,81$.

[3] Kramers H. A. et Heisenberg W. - Z. Physik, 1925, 31, $68 \mathrm{r}$.
[4] Hanle W. - Naturwiss., $1931,19,375$.

BÄr R. - Naturwiss., I $93 \mathrm{r}, \mathbf{1 9}, 463$.

Kastler A. - J. Physique Rad., i933, 4, 406. 